\title{
Application of Customer Analysis Methods to Online Business Services- Take Pinduoduo for Example
}

\author{
Zhixi Zhou
}

Intensive English Program, University of Miami, USA

Corresponding author's e-mail:Vivian.wang@cas-harbour.org

\begin{abstract}
With the development of science and technology, online shopping is becoming more and more popular, especially in the populous China. Faced with such fierce competition in the online e-commerce market, Pinduoduo had gained growing attention among the two e-commerce giants Taobao and JD.com at that time. Most of the data for the study came from articles in ScienceDirect, Walkthechat and the Love of Academia. With the help of customer analysis methods, this paper analyzes Pinduoduo's current customers demographics and pinpoints potential target audience. The audience groups that the company lacks and puts forward suggestions. This paper discovers that the majority of Pinduoduo users are citizens with low education levels who live in cities above tier 3 in China. In terms of its operational bottlenecks, Pinduoduo is the product of a consumer downgrade, which means Pinduoduo appeals to customers who emphasize solely on price instead of product quality, which encourages the selling of counterfeits, entailing negative impact on the brand image. In 2018, Pinduoduo was also accused of selling counterfeits with substandard quality. Therefore, Pinduoduo needs to have a severe punishment mechanism and open a high-end commodity entrance and cooperate with well-known brands in order to strengthen its audience base, average order price and product quality. Adopting this strategy Pinduoduo can potentially improve its competitive advantage and secure a bigger market share in Chinese e-commerce market.
\end{abstract}

Keywords: Pinduoduo, Customer analysis method, Data, Suggestions

\section{INTRODUCTION}

China is a $\$ 1.94$ trillion e-commerce market, the largest in the world that triples the market size in the U.S. [8]. However, this is also a market which is hard to enter. Pinduoduo is a social e-commerce company founded by Shanghai Dream Information Technology Co., Ltd. in September 2015 with more than 200 million users in 2018. Pinduoduo has quickly gained a foothold in the ecommerce market with the advantage of hard covering its target consumer group. However, on June 7, 2018, the legal Evening News, a daily newspaper, claimed that "Pinduoduo now sells illegal products." In February of the same year, The China Consumers' Association issued a report on Online Shopping, Price, Quality and Aftersales Service on the "Singles' Day" of 2017, "which clearly pointed out that some goods sold on Pinduoduo platforms were fake and substandard in quality [1]. Therefore, it has a serious impact on its brand image. In this report, customer analysis methods, such as statistical analysis, will be used to analyze Pinduoduo customers' education level and socioeconomic status. Moreover, a competitor landscape analysis will also be conducted to analyze the advantages and disadvantages of Pinduoduo. In addition, this report will propose recommendations that could help Pinduoduo strengthen its market presence.

\section{STATISTICAL ANALYSIS}

Common customer analysis techniques include observation, experiment, experience summary and statistical analysis [2]. An introduction to the methods used in this article is statistical analysis. Statistical analysis is the use of statistical principles, through the collection, collation and analysis of the network business data, a quantitative research method [2]. 


\section{APPLICATION OF STATISTICAL ANALYSIS IN E-COMMERCE SERVICE}

\subsection{Statistical analysis of Pinduoduo users' education level}

\subsubsection{Statistics on users' education level}

The educational level of Pinduoduo and Taobao users was counted, and the results were shown in figure 1 .

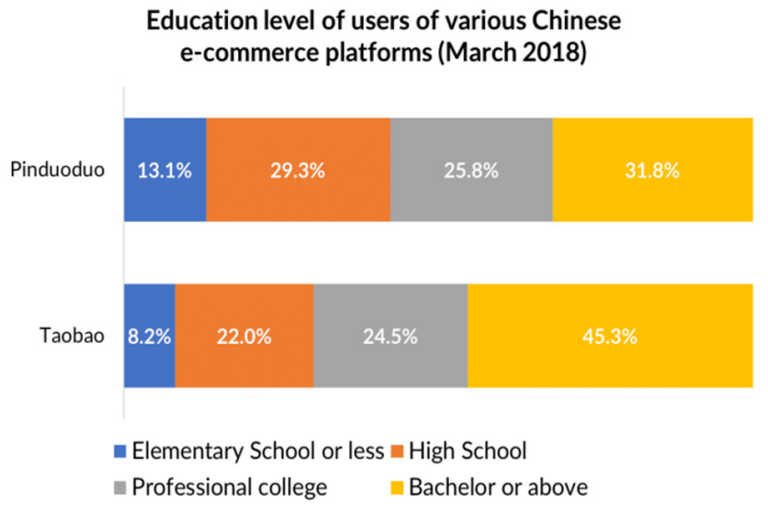

Figure 1 Education level of users of various Chinese ecommerce platforms [4]

As can be seen from figure 1 , nearly 70 percent of Pinduoduo's customers have a Bachelor degree or below. And nearly half of Taobao's customers are Bachelor and above. And nearly half of Pinduoduo's customers don't go to Professional college. Therefore, the education level of Pinduoduo users is low.

\subsubsection{Analysis on the reasons for the statistics}

According to the result (1), it is evident that Pinduoduo users have lower education levels; meanwhile, the education level has an effect on the consumer market. Because of their weak brand recognition ability, it is difficult for them to distinguish the quality of products and realize the potential harm of buying substandard goods. Thus, they may not know most well-known brands and have relatively lower brand loyalty [3]. Therefore, they do not really pay more for brand value. Whether it is an "iPhone" or an "ePhone," they will buy an "ePhone" instead of an "iPhone" as long as it works, has the same functionality, and is cheaper. However, highly educated consumers have brand awareness [3]. Therefore, this is why there is only a small amount of Bachelor among Pinduoduo users. By contrast, the illegal act of selling cheap fakes and low standard products is certainly not a long-term solution. Consequently, Pinduoduo needs to strengthen supervision and cooperate with brands to get more authentic sales channels. Such an approach would not only improve the company's credibility, but also attract more highly educated consumers.

\subsection{Statistical analysis of residential city rank of Pinduoduo users}

\subsubsection{The statistics of users' living city level}

The residential city tires of Pinduoduo, Taobao, JD.com, Tmall and Vip.com users were statistically analyzed, and the results were shown in figure 2 .

City distribution of users of various Chinese e-commerce platforms (March 2018)

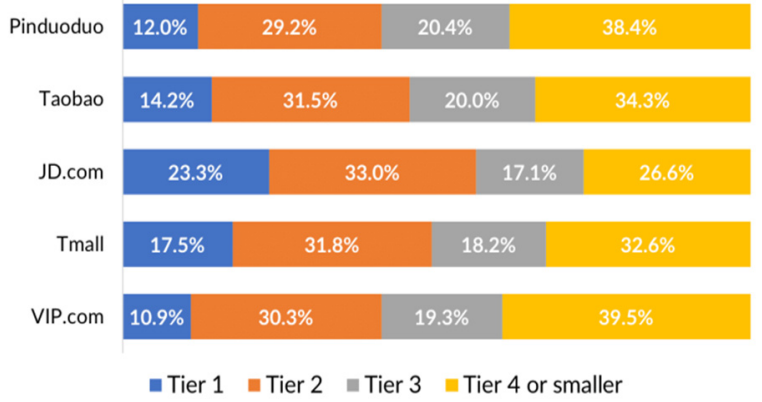

Figure 2 City distribution of users of various Chinese ecommerce platforms [4]

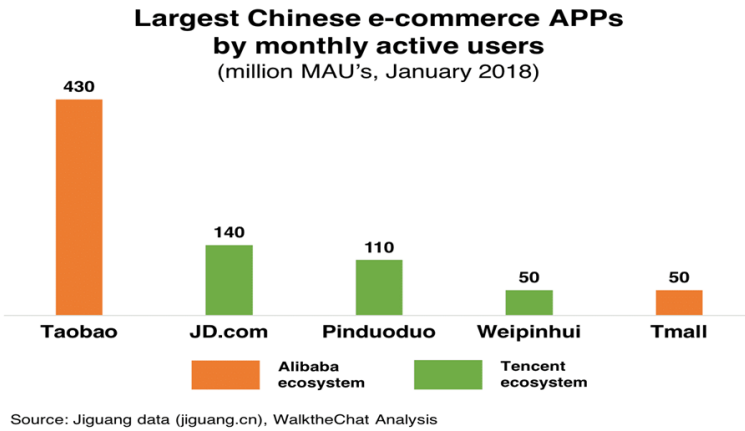

Figure 3 Largest Chinese e-commerce Apps by monthly active users [5]

Figure 3 shows that Taobao, JD.com and Pinduoduo are the most active e-commerce apps in China. Figure 2 shows that Pinduoduo living in first-tier cities have $12 \%$, $14.2 \%$ and $23.3 \%$ users on Taobao and JD.com respectively. This suggests that there are fewer people in Shanghai, Beijing and Guangzhou, which are more developed parts of China. Moreover, there are 4.5 percent fewer Pinduoduo users living in first - and second-tier cities than Taobao and 15.1 percent fewer JD.com users. Based on the data in Figure 3, this is actually a large number of people. In terms of a company, it needs these people to enter its market.

\subsubsection{Analysis on the reasons for the statistics}

It can be concluded from (3) that most Pinduoduo users are residents living in cities below tire 3 . This is due to two factors. First, it is due to patchwork parity strategies, such as "nine yuan nine limited second kill" campaigns. Moreover, mass consumption in daily life is 
a downward trend in consumption. For consumers, they are more concerned about price than quality. As a result, poorer residents of China's lower-tier cities will be attracted by the price tag [3]. Second, the consumption downgrade is a social exchange in which the market is flooded with substandard products. As a result, goods sold at Pinduoduo are perceived as cheap with poor quality. With the development of the economy, their hearts are stronger and they are more knowledgeable, so they will pay more attention to the quality of life. That means they like better value for money and better-quality products. Because of their social status, they will also buy some products with high brand value or even premium price because of their face. This is face consumption - consuming products to enhance and maintain your face [6]. Thus, that is why most people in first-tier and second-tier cities rarely buy the products Pinduoduo sells. In this way, Pinduoduo needs to sell some cost-effective and renown products.

\subsection{Application of statistical analysis conclusions}

\subsubsection{Strengthen supervision}

As mentioned earlier in this report, Pinduoduo has a lot of problems with selling counterfeit goods. Therefore, the Pinduoduo platform itself needs to strengthen supervision. First, the Pinduoduo platform requires each merchant to provide his or her own identity certificate, such as an ID card, and the platform needs to check, after which the merchant can sell goods. Second, Pinduoduo needs to give every merchant a credit rating. There are 5 grades, from best to worst: excellent, good, average, unrated and poor. The best sellers need to sell 100 items on Pinduoduo without fakes. A good seller needs to sell 50 items on Pinduoduo without fakes. To get the average merchant needs to sell 25 items on Pinduoduo without fakes. Businesses that did not sell 25 but did not sell counterfeit items were rated unrated. No matter how many products are sold, only those who have sold a fake once are rated as poor. Third, the mechanism to punish the sellers of counterfeit goods. Third, the mechanism to punish the sellers of counterfeit goods. In addition to having a poor credit rating, first-time sellers will have to refund the amount of goods sold plus a tenth of that amount to the buyer. For example, a buyer who buys a $\$ 100$ piece of clothing reports the seller for selling fake goods and doing so. The merchant needs to refund $\$ 110$ to the buyer. If the merchant wants to go back to unrated, it needs to sell 50 genuine items. The second time, the seller will be directly removed from Pinduoduo and the person on the ID card will never be able to open another shop at Pinduoduo.

\subsubsection{JD.com's strategy for expanding its consumer space}

As can be seen from Figure 2, only $43.7 \%$ of JD.con users live in cities at or below tier 3 . That was 10.6 percent less than the number of Taobao users living in the third line or below. Similarly, the number of users in the third line and below was $15.1 \%$ lower than that of Pinduoduo. Thus, in order to get more people living in smaller cities to buy on the platform, Jd.com has invested heavily in promotions. For example, JD.com made a change in its 618-year promotion (named after the date June 18). Most brands are taking part in a $\$ 50$ discount for every $\$ 400$ shopping cart. For certain categories of goods, JD.com offers an extra 300-yuan coupon that can be used on top of other discounts [7]. JD.com's approach is clearly a success. According to data displayed by JD.com, its GMV (Gross Merchandise Volume) during the 618,2019 shopping festival increased by $27 \%$ compared to GMV in 2018. Moreover, JD.com recorded 88 million DAU (Daily Active Users) in the 618shopping festival, up $20 \%$ year on year. For products with high requirements, such as milk and milk powder, JD.com sold 350 million cartons of milk and 10 million cans of milk powder in the 618-shopping festival in 2019 (WalktheChat.com). In addition, the GMV growth rate of fresh such products reached $90 \%$ year-on-year in 618 days.

\subsubsection{A suggestion for Pinduoduo to enhance competitiveness}

According to (2) and (4), Pinduoduo needs to sell products of relatively well-known brands with high quality. Therefore, Pinduoduo needs the interface to open a high-price quality area, just as JD.com and Taobao also adopt group-buying and open a low-price area in order to broaden their audience. The most important thing Pinduoduo needs to do is to cooperate with renowned brands in the high-priced area and to guarantee the quality and authenticity of products. A recommendation for Pinduoduo needs to be to cooperate with the big brands in the high-priced area and to guarantee the quality and authentic products. The big brands here are not luxury brands such as Louis Vuitton, but well-known and practical household brands such as Nestle coffee, Jule milk and so on. It can be concluded from (6) that there is a great demand for commodities of relatively low price and good quality. Therefore, Pinduoduo can use its strength in Numbers to negotiate lower prices with major commodity companies. For example, the Pinduoduo platform will subsidize how much a company sells its products and how much it gets a discount for buying those products. In this way, richer people in first-tier and second-tier cities will also spend money because Pinduoduo's high-priced districts sell products with high cost performance, high brand awareness and guaranteed quality. That way, Pinduoduo won't be labelled as cheap 
but of poor quality. Richer people in first-tier and second-tier cities also spend money because Pinduoduo's high-priced districts sell products with better price performance, better brand awareness and guaranteed quality. Furthermore, they will not be deterred from buying products sold on Pinduoduo because of their face.

\section{CONCLUSION}

According to my result, Pinduoduo has learned about the loss of the company's audience, so it is suggested that Pinduoduo cooperate with good brands to sell costeffective products. Thus, people with high education or living in first-tier and second-tier cities can buy the products sold on its platform. In this way, Pinduoduo will gain good economic benefits, super competitive advantage in the market, and the sustainability of its business model will not be challenged. Second, Pinduoduo's adoption of my punishment mechanism for selling counterfeits should make the situation of Pinduoduo selling counterfeits better. Thus, let more people trust and use it.

There are two potential biases with this report. First, the data are from 2018 and 2019, which may be out of date in the e-commerce market where the market changes a lot with the market developing at a staggering rate. Second, JD.com is looking for the low-end market, while Pinduoduo needs the high-end market. Since China's low-end market has greater potential, this example may not be appropriate. This article is also useful, because the epidemic in 2020 , basically the major companies have not changed. Pinduoduo still does not open high-end merchandise entries on the page. Therefore, the part that can be changed in this article is, first, to find the Pinduoduo 2020 data from other organizations to ensure that there is no big change this year. Second, it is also practical to find a brand by using high-end brands to perfect the defects of their audience groups in order to find the effectiveness of the high-end market or to use the virtual market to make sure the effect.

\section{REFERENCES}

[1] Q. Fan, Research on precision marketing strategy and Optimization of Pinduoduo, Journal of Tianjin Vocational College of Commerce, Vol. 8, No. 1, 2020, pp. 61-65

[2] C. Liu, T. Zhang. Application of customer analysis technology in hotel service, Times Economy and Trade, No. 235, March 2012, pp. 41-42

[3] C. Fang, H. Jiang. China's economic and social development from the perspective of Pinduoduo's listing, National Circulation Economy, 2018, ecommerce section, pp. 18-19
[4] T. Graziani. Pinduoduo: A close look at the fastest growing e-commerce app in China, WalktheChat (2018, August 27), QuestMobile, 2018.3

[5] T. Graziani. Pinduoduo: A close look at the fastest growing e-commerce app in China, WalktheChat (2018, August 27), Jiguang data (jiguang.cn)

[6] J.J. Li, C. Su. How face influences consumption: A comparative study of American and Chinese consumers, International Journal of Market Research, 49 (2) (2007), pp. 237-256

[7] T. Graziani. 618 Sales Data 2019: Tall growth slows down; Pinduoduo traffic up 48\%, WalktheChat (2019, June 30)

[8] A. Lipsman. Global Ecommerce 2019: Ecommerce Continues Strong Gains Amid Global Economic Uncertainty, eMarketer (2019, June 27) 